\title{
Cannabis Abstinence During Treatment and One-Year Follow-Up: Relationship to Neural Activity in Men
}

\author{
Hedy Kober',3, Elise E DeVito',3, Cameron M DeLeone', Kathleen M Carroll' and Marc N Potenza',2 \\ 'Department of Psychiatry, Yale University, New Haven, CT, USA; ${ }^{2}$ Departments of Neurobiology and Child Study Center, Yale University, \\ New Haven, CT, USA
}

\begin{abstract}
Cannabis is among the most frequently abused substances in the United States. Cognitive control is a contributory factor in the maintenance of substance-use disorders and may relate to treatment response. Therefore, we assessed whether cognitive-controlrelated neural activity before treatment differs between treatment-seeking cannabis-dependent and healthy individuals and relates to cannabis-abstinence measures during treatment and I-year follow-up. Cannabis-dependent males $(N=20)$ completed a functional magnetic resonance imaging ( $\mathrm{fMRI}$ ) cognitive-control (Stroop) task before a I2-week randomized controlled trial of cognitive-behavioral therapy and/or contingency management. A healthy-comparison group $(N=20)$ also completed the $f$ MRI task. Cannabis use was assessed by urine toxicology and self-report during treatment, and by self-report across a I-year follow-up period ( $N=18)$. The cannabis-dependent group displayed diminished Stroop-related neural activity relative to the healthy-comparison group in multiple regions, including those strongly implicated in cognitive-control and addiction-related processes (eg, dorsolateral prefrontal cortex and ventral striatum). The groups did not differ significantly in response times (cannabis-dependent, $N=12$; healthy-comparison, $N=14$ ). Within the cannabis-dependent group, greater Stroop-related activity in regions including the dorsal anterior cingulate cortex was associated with less cannabis use during treatment. Greater activity in regions including the ventral striatum was associated with less cannabis use during I-year posttreatment follow-up. These data suggest that lower cognitive-control-related neural activity in classic 'control' regions (eg, dorsolateral prefrontal cortex and dorsal anterior cingulate) and classic 'salience/reward/learning' regions (eg, ventral striatum) differentiates cannabis-dependent individuals from healthy individuals and relates to less abstinence within-treatment and during long-term follow-up. Clinically, results suggest that treatment development efforts that focus on enhancing cognitive control in addition to abstinence may improve treatment outcomes in cannabis dependence.
\end{abstract}

Neuropsychopharmacology (2014) 39, 2288-2298; doi:I0.1038/npp.2014.82; published online I8 June 20I4

\section{INTRODUCTION}

Cannabis is a commonly abused drug in the United States (SAMHSA, 2011). In 2010, of an estimated 17.4 million current cannabis users, 4.5 million $(\sim 26 \%)$ qualified for cannabis-use disorders (SAMHSA, 2011). The most studied and promising treatments include cognitive-behavioral therapy, contingency management or a combination thereof (Budney et al, 2007). However, most treatment recipients do not achieve prolonged abstinence; even well-validated therapies achieve abstinence rates of $\sim 50 \%$ during, and $25 \% 1$ year following, treatment (Budney et al, 2007). One promising strategy for enhancing treatment efficacy involves improving our understanding of how cognitive and neural function may be disrupted in cannabis-related disorders, and how such disruptions may be related to treatment outcome (Potenza et al, 2011).

\footnotetext{
*Correspondence: Dr H Kober, Department of Psychiatry, Yale University, I Church Street, Suite 70I, New Haven, CT 065I0, USA, Tel: + I 203737 564I, Fax: + 203737 359I, E-mail: hedy.kober@yale.edu ${ }^{3}$ These authors contributed equally to this work.

Received 26 November 2013; revised 24 March 2014; accepted 25 March 2014; accepted article preview online 7 April 2014
}

Cognitive control, conceptualized as 'the ability to orchestrate thought and action in accordance with internal goals' (Miller and Cohen, 2001), is implicated in addiction vulnerability and maintenance (Carpenter et al, 2006; Peterson et al, 1999; Smith et al, 2013). Cognitive control includes constituent processes such as goal maintenance, selective attention, conflict monitoring and resolution, and response inhibition, which may be implemented in different subregions of prefrontal cortex (eg, dorsal anterior cingulate cortex and error detection; dorsolateral prefrontal cortex and control implementation; Miller and Cohen, 2001; Peterson et al, 1999). These cognitive constructs are relevant to core clinical characteristics of addiction, including enhanced allocation of attentional resources toward drug-related cues, and diminished control over drug craving and drug taking (Carpenter et al, 2006; Peterson et al, 1999).

Neuropsychological and neuroimaging studies have provided evidence for disruption in several cognitive processes in chronic cannabis users, including cognitive control. For example, chronic cannabis users perform less well than healthy peers on measures of memory, information processing, and cognitive control (eg, Schreiner and Dunn, 2012; Solowij et al, 2002, but also see Takagi et al, 
2011). Further, compared with non-drug-using adults, cannabis users often exhibit lower neural activity during performance on memory, learning, and cognitive-control tasks (including the Stroop color-word interference task) across several brain regions, including prefrontal cortex (Battisti et al, 2010; Eldreth et al, 2004; Gruber and Yurgelun-Todd, 2005, but c.f., Harding et al, 2012).

In addition to its proposed general role in addiction, cognitive control may be important for treatment engagement and success (DeVito et al, 2012; Potenza et al, 2011; Streeter et al, 2008), particularly with more cognitively demanding therapies such as cognitive-behavioral therapy (Carroll, 1998; Carroll et al, 2011). Such treatments may work (at least in part) by enhancing control over drug craving and drug taking (Kober et al, 2010). Consistently, cognitive control, as measured by Stroop task performance, predicted treatment compliance and retention in cocainedependent individuals (Streeter et al, 2008). Similarly, pretreatment neural activity during cognitive-control tasks, such as Stroop, correlated with within-treatment abstinence in cocaine-dependent individuals (Brewer et al, 2008) and moderated associations between craving and subsequent smoking in nicotine-dependent individuals (Berkman et al, 2011). In both studies, greater pretreatment activity in prefrontal regions, including anterior cingulate and ventrolateral prefrontal cortex, was associated with better treatment outcome. Lower functional activity in a 'cognitive-control' network (eg, dorsolateral prefrontal cortex) was associated with more impulsive choice on a delay discounting paradigm (Stanger et al, 2013), and impulsive choice had been previously been associated with poorer treatment outcomes adolescent cannabis users (Stanger et al, 2012). Comparing pre- with posttreatment neural activity, we previously reported decreased Strooprelated neural activity following behavioral drug treatment in a mixed group of substance users, suggesting that treatment is associated with increased efficiency in cognitive-control circuits (DeVito et al, 2012). Taken together, these findings suggest that cognitive control may be a contributory factor to substance-use initiation and maintenance, as well as in treatment response.

However, whether cognitive-control-related neural activity is associated with within-treatment and posttreatment abstinence outcomes has not been concurrently assessed in any substance-using populations, including cannabis dependence. Both within-treatment and posttreatment abstinence are important to assess because they represent clinically distinct phases of treatment, with different challenges and contextual conditions. For example, within treatment, participants have access to structured support, are working to change established habits, and initial abstinence is associated with acute withdrawal symptoms. However, after patients leave treatment, structured support is less available, participants are working to maintain new habits, and withdrawal symptoms are less salient after prolonged abstinence. These and other factors likely influence behavioral and neural mechanisms underlying ongoing abstinence in each stage.

This study aims to address these gaps in knowledge by using a cognitive-control (Stroop) task during functional magnetic resonance imaging (fMRI) to investigate: (1) differences in Stroop-related neural activity between pretreatment cannabis-dependent males and healthycomparison individuals; and, (2) how pretreatment neural activity relates to cannabis abstinence within-treatment and 1-year post treatment. We hypothesized that: cannabisdependent individuals would exhibit lower Stroop-related neural activity relative to healthy-comparison individuals in regions previously associated with cognitive control, including anterior cingulate, dorsolateral prefrontal cortex and striatum (Brewer et al, 2008; Eldreth et al, 2004; Gruber and Yurgelun-Todd, 2005). Within cannabis-dependent individuals, we hypothesized that pretreatment neural activity would be associated with treatment outcome, such that individuals with more normative pretreatment Strooprelated activity would use less cannabis during treatment and at 1-year follow-up.

\section{MATERIALS AND METHODS}

\section{Participants}

Twenty cannabis-dependent individuals and 20 matched healthy non-drug-using individuals participated in this fMRI study and were all included in fMRI analyses. Cannabis-dependent individuals were recruited before beginning treatment in a randomized controlled trial (RCT; Carroll et al, 2012). RCT participants were Englishspeaking adults who met current cannabis-dependence criteria as determined by the Structured Clinical Interview for DSM-IV (SCID; First et al, 1996), used cannabis in the previous 28 days, and committed to completing 12 weeks of outpatient treatment (cognitive-behavioral therapy and/or contingency management; see Supplementary Materials). Patients were offered participation in the fMRI component if they reported no claustrophobia, colorblindness, history of severe head trauma with loss of consciousness, neurological disorders, or MRI-contraindicated metallic implants. fMRI scanning was conducted immediately before treatment initiation.

Age- and sex-matched healthy- comparison community participants were recruited using advertisements. Healthycomparison participants were additionally excluded for SCID-determined lifetime Axis-I psychiatric disorders, including substance abuse or dependence (except nicotine dependence), and recent psychotropic medication use. After complete study description, subjects provided written informed consent in accordance with Yale's Institutional Review Board.

\section{Clinical Assessments}

At pretreatment, IQ was estimated by the Shipley Institute of Living Scale (Zachary, 1991) and the Addiction Severity Index assessed self-reported lifetime and past-28-day substance use (Table 1; Carroll et al, 2012). Weekly within-treatment substance-use assessments included selfreported past-week use with a Timeline Follow-back method (Robinson et al, 2012; Sobell and Sobell, 1992) and urine toxicology screening. Posttreatment follow-up appointments were scheduled at 3-month intervals for 1 year to collect urinalysis and Timeline Follow-back of selfreported use since last visit. Our long-term follow-up estimate of drug use was calculated as percent of 


\section{Cannabis-treatment outcomes and fMRI}

$\mathrm{H}$ Kober et al

Table I Demographics, Treatment Engagement, and Clinical Outcomes

Participant groups

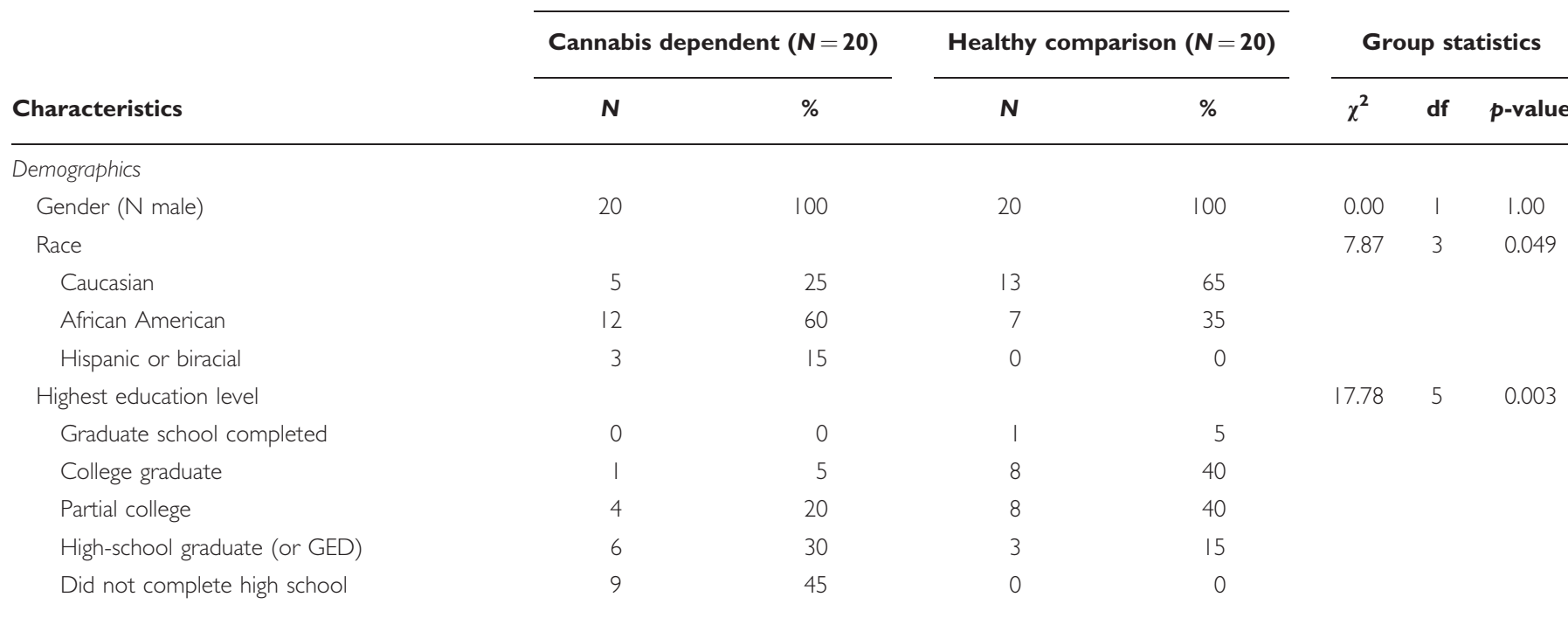

Baseline clinical and substance-use measures

Psychiatric diagnosis

Lifetime major depression

Current major depression

Lifetime anxiety disorder

Antisocial personality disorder

Lifetime alcohol-use disorder

Current alcohol abuse

Daily cigarette smoker

Treatment admission prompted by legal system

$\begin{array}{ll}2 & 10 \\ 1 \\ 1 \\ 5 \\ 8 \\ 1 \\ 11 \\ 19\end{array}$

\begin{tabular}{|c|c|c|c|c|c|c|c|}
\hline & Mean & SD & Mean & SD & $T$ & df & p-value \\
\hline \multicolumn{8}{|l|}{ Demographics } \\
\hline Age (years) & 26.65 & 9.81 & 29.20 & 10.06 & 0.81 & 38 & 0.42 \\
\hline Estimated IQ (Shipley Institute of Living Scale) & 93.10 & 12.80 & 108.42 & 10.30 & 4.11 & 37 & $<0.001$ \\
\hline
\end{tabular}

Baseline clinical and substance-use measures ${ }^{a}$

Days use in 28 days before treatment

$\begin{array}{lrr}\text { Cannabis } & 17.55 & 9.93 \\ \text { Cigarette } & 17.60 & 12.63 \\ \text { Alcohol } & 3.90 & 4.75 \\ \text { Lifetime years of substance use } & & \\ \text { Cannabis } & 12.40 & 10.32 \\ \text { Alcohol } & 10.75 & 9.06 \\ \text { Lifetime number of arrests } & 5.75 & 4.02\end{array}$

Treatment engagement and outcomes ${ }^{b}$

Days in Treatment

$60.10 \quad 28.57$

Cannabis use within treatment

$\begin{array}{lll}\text { \% Negative urines } & 33.72 & 35.63 \\ \text { Maximum self-reported consecutive days abstinence } & 39.60 & 30.59 \\ \text { \% Days self-reported abstinence } & 62.50 & 40.44\end{array}$

Cannabis use during follow-up ${ }^{c}$

No. of follow-up sessions attended 
Table I (Continued)

\begin{tabular}{|c|c|c|c|c|c|c|c|}
\hline & Mean & SD & Mean & SD & $T$ & df & $p$-value \\
\hline Days of follow-up information collected & $3 \mid 1.11$ & 98.36 & & & & & \\
\hline$\%$ Days self-reported abstinence during follow-up & 57.05 & 38.65 & & & & & \\
\hline \multicolumn{8}{|l|}{ Stroop behavioral measures ${ }^{d}$} \\
\hline Congruent trial response-time (ms) & 584 & 82 & 590 & 75 & 0.18 & 24 & 0.86 \\
\hline Incongruent trial response-time (ms) & 785 & 150 & 780 & 148 & 0.07 & 24 & 0.94 \\
\hline No. of errors during incongruent trials & 10.17 & 8 & 7.14 & 5.75 & 1.12 & 24 & 0.27 \\
\hline
\end{tabular}

asome clinical data, including past month days of cigarette and alcohol-use measures, were not available for the healthy-control group.

${ }^{\mathrm{b}}$ Cannabis-abstinence information is also described in Supplementary Table S3.

${ }^{c}$ I-year follow-up data were only available for 18 subjects.

${ }^{\mathrm{d}}$ Stroop behavioral data were only available for 12 cannabis-dependent and 14 healthy-comparison participants. The remaining behavioral data were not available because of technical failures during data collection.

participant-reported abstinent days from total days of available follow-up data (Table 1). Within-treatment and follow-up variables were included in correlation analyses with neural activity.

\section{Stroop Color-Word Interference Task}

Participants were administered an event-related fMRI Stroop color-word interference task (MacLeod, 1991) wherein color words are presented in congruent ('RED' in red ink) or incongruent ('RED' in blue ink) pairs. Correctly naming incongruent ink colors requires several components of cognitive control, including selective attention, conflict monitoring and resolution, and response inhibition (Carpenter et al, 2006). As previously described (DeVito et al, 2012), participants performed six runs. On each trial, participants were asked to silently name the ink color of a congruent or incongruent color-word. Each run consisted of 105 trials, wherein each word was presented for $1300 \mathrm{~ms}$, with an inter-trial interval of $350 \mathrm{~ms}$. Each run included seven incongruent events, presented pseudo-randomly every 13-16 congruent stimuli. The task was administered via back-screen projection using Presentation software (Neurobehavioral Systems, Albany, CA). Participants completed two pre-scan Stroop practice runs and five post-scan runs during which response times were recorded. To assess group differences in Stroop Effect (ie, slower reaction times to incongruent $v s$ congruent color-word pairs), we ran mixed ANOVAs including trial type (congruent and incongruent) as a within-group and diagnostic group (cannabisdependent and healthy-comparison) as a between-group factor. We used $t$-tests to compare incongruent trial error rates between diagnostic groups (Table 1). Owing to technical difficulties, reliable response-time data were not available for 14 subjects (8 cannabis-dependent and 6 healthy-comparison). Cannabis-dependent participants for whom response-time data were available did not differ in demographics, clinical characteristics, or treatment outcome from those without available data. In addition, the restricted samples of cannabis-dependent $(N=12)$ and healthy-comparison $(N=14)$ groups with response-time data remained matched for age.

\section{fMRI Data Acquisition, Preprocessing and Analysis}

After acquisition, data were preprocessed in SPM5 as previously described (DeVito et al, 2012; Kober et al, 2010); for details, see Supplementary Materials). First-level robust regression was implemented in MATLAB 7.3 (Mathworks, Natick, MA), using the standard general linear model but with iteratively reweighted least squares using the bisquare weighting function to reduce the effects of outliers (Wager et al, 2005). Motion parameters and high-pass filter parameters were regressors of no interest. Subsequently, we performed a second-level, random-effects analysis to compare activity between groups, using NeuroElf (NeuroElf. net). To characterize Stroop-related neural activity, we first compared activity in incongruent $v s$ congruent trials (incongruent > congruent; 'Stroop effect') within and between cannabis-dependent and healthy-comparison groups. Results were family-wise-error corrected at $p<0.05$.

To assess the relationship between within-treatment cannabis use and brain activity, we computed three whole-brain robust correlations between pretreatment Stroop-related neural activity and three a priori clinical indicators of cannabis use (Carroll et al, 2012; DeVito et al, 2012; Kober et al, 2010). Indicators were chosen for their frequent use in the RCT literature and demonstrated relationship to shorter- and longer-term outcomes across studies (Budney et al, 2007; Carroll et al, 2012; MTP Research Group, 2004; Peters et al, 2011). Within-treatment indicators were percent cannabis-negative urines, percent self-reported days abstinent, and self-reported maximum consecutive days abstinent. The three correlation maps were then used in conjunction analyses to identify regions commonly correlated with all three within-treatment outcome variables. Each correlation map was initially thresholded at $p<0.05$ (two-tailed), and formal conjunction logic was applied (absolute values of map ${ }^{*}$ map $2^{\star}$ map3), resulting in a map that identified voxels significant in all three maps-at a combined conjunction threshold of $p<0.000125$, plus an added cluster threshold of 20 contiguous voxels $(k=20)$. To assess the relationship between brain activity and cannabis use over longer-term follow-up, we computed whole-brain robust correlations between pretreatment Stroop-related neural activity and 
self-reported percent days abstinent from cannabis across the 1-year posttreatment period $(N=18$; Supplementary Materials). Results were family-wise-error corrected at $p<0.05$.

\section{RESULTS}

\section{Baseline Differences between Cannabis Users and Comparison Subjects}

Demographics. Although both males and females were invited to participate, the RCT sample was predominantly male, and only one female was both eligible and interested in fMRI participation. Given concerns regarding possible sex-related differences, this female was excluded. The resulting all-male groups did not differ in age $t_{(38)}=0.81$, $p=0.42)$. Healthy-comparison individuals had a higher average IQ $\left(t_{(37)}=4.12, p<0.001\right)$, and more cannabisdependent individuals reported daily cigarette smoking $\left(\chi_{(1,38)}^{2}=14.55, p<0.001\right)$, and the groups were not matched on race/ethnicity (Table 1 ). Within the cannabisdependent group, demographic and clinical characteristics were consistent with those of the overall RCT sample (Supplementary Table S1; Carroll et al, 2012).

Response times. A robust Stroop effect was observed across both groups, with slower response times for incongruent than congruent trials $\left(\mathrm{F}_{(1,24)}=113.89, p<0.001\right)$. Groups did not differ in overall response times $\left(\mathrm{F}_{(1,24)}=0.00, p=0.99\right)$, magnitude of Stroop effect $\left(\mathrm{F}_{(1,24)}=0.07, p=0.79\right)$ or number of errors $\left(t_{(24)}=1.12\right.$, $p=0.27$; Table 1$)$. As expected, both groups displayed evidence of a speed-accuracy trade off, with faster RTs associated with more errors (Supplementary Materials; Supplementary Table S2; Supplementary Figures S1-S10).

fMRI results. Within each group, patterns of activity were broadly consistent with previous fMRI Stroop studies (eg, DeVito et al, 2012); Supplementary Figures S1-S4). Between-group comparison revealed greater Stroop-related activity in healthy-comparison individuals relative to cannabis-dependent individuals in several prefrontal regions, including bilateral dorsolateral and dorsal anterior prefrontal cortex, as well as in ventral and dorsal striatum (caudate), amygdala/parahippocampal gyrus, thalamus, and midbrain regions, including the ventral tegmental area and substantia nigra, right insula/superior temporal gyrus, and right posterior superior/middle temporal gyrus (Table 2; Figure 1; Supplementary Figures S1 and S4). Data extracted from these regions indicated that these effects were driven by increased activity during incongruent trials in healthycomparison individuals relative to cannabis-dependent individuals, with similar activity across groups during congruent trials (Figure 1).

\section{Relationship between Pretreatment fMRI and Within-Treatment Cannabis-Use Outcomes}

The conjunction analysis identified positive correlations (between Stroop-related neural activity and all three withintreatment cannabis-use variables) in regions including dorsal anterior cingulate, cuneus, posterior cingulate, and lingual and supramarginal gyri bilaterally (Table 2; Figure 2; Supplementary Figures S6-S8). Positive correlations indicate that greater Stroop-related activity was associated with better abstinence outcomes. These regions overlap with the regions showing the Stroop effect in the cannabis-dependent group (Supplementary Figures S1 and S3).

\section{Relationship between PreTreatment fMRI and 1-Year Follow-Up}

Robust whole-brain correlations identified several brain regions where Stroop-related activity positively correlated with cannabis abstinence across 1-year follow-up, including ventral striatum, posterior cingulate, bilateral middle/ inferior occipital gyri, cerebellum, and prefrontal regions including ventrolateral prefrontal cortex and perigenual/ subgenual anterior cingulate (Figure 3, Supplementary Figure S4; Table 2). Greater Stroop-related activity was associated with better treatment outcome. We observed a negative correlation in a single region encompassing the left insula and lentiform nucleus and extending into inferior frontal gyrus. Again, these regions overlap with the regions showing the Stroop effect in the cannabis-dependent group (Supplementary Figures S1 and S3).

\section{DISCUSSION}

To our knowledge, this is the first study to assess the relationship between pretreatment neural activity and treatment outcome in cannabis dependence, and the first to investigate this relationship with both within-treatment and longer-term posttreatment abstinence in any substancedependent population. As predicted, cannabis-dependent relative to healthy-comparison individuals exhibited diminished Stroop-related activation in regions including dorsolateral prefrontal cortex and ventral striatum. Within the cannabis-dependent group, greater pretreatment Strooprelated activation was generally associated with better outcomes, operationalized as lower rates of cannabis use within treatment and across 1-year follow-up. Notably, regions of Stroop-related activation that correlated with all three within-treatment abstinence measures (eg, dorsal anterior cingulate) were different from those that correlated with posttreatment abstinence rates (eg, ventral striatum). This is consistent with the idea that different cognitivecontrol processes may underlie within- $v s$ posttreatment abstinence.

\section{Differences between Cannabis-Dependent and Healthy-Comparison Groups}

Although some of the response-time data were missing, the available data suggest that groups did not differ on Stroop behavioral measures (consistent with prior findings, eg, Eldreth et al, 2004); cannabis-dependent relative to healthycomparison individuals demonstrated lower Stroop-related neural activity in dorsal prefrontal cortex and dorsal and ventral striatum, amygdale, and parahippocampal gyrus. These results are broadly consistent with prior findings from a blocked-variant Stroop task, in which relatively diminished activation was reported in cannabis users in regions including the anterior cingulate and dorsolateral 
Table 2 Stroop-Related Neural Activity in Cannabis-Dependent and Healthy-Comparison Individuals: Group Differences and Relationship to Treatment

\begin{tabular}{|c|c|c|c|c|c|c|c|}
\hline \multirow[b]{2}{*}{ Regions of activation } & \multirow[b]{2}{*}{ Laterality } & \multicolumn{3}{|c|}{ Peak coordinates } & \multirow[b]{2}{*}{ Cluster size } & \multicolumn{2}{|c|}{ Statistics } \\
\hline & & $x$ & $y$ & $\mathbf{z}$ & & Maximum & Mean \\
\hline \multicolumn{8}{|l|}{ Group differences in Stroop-related neural activity at pretreatment baseline ${ }^{a}$ : } \\
\hline Healthy comparisons > cannabis dependent & & & & & & $\mathbf{t}$ & $\mathbf{t}$ \\
\hline $\begin{array}{l}\text { Ventral Striatum/dorsal striatum/midbrain/subthalamic nucleus/thalamus/ } \\
\text { amygdala/parahippocampal gyrus }\end{array}$ & $R+L$ & -12 & -12 & -9 & 674 & -4.56 & -2.45 \\
\hline Dorsolateral prefrontal cortex/middle frontal gyrus & $\mathrm{L}$ & -54 & 15 & 39 & 164 & -4.01 & -2.59 \\
\hline Dorsal prefrontal cortex/superior frontal gyrus & $\mathrm{R}$ & 24 & 45 & 33 & 147 & -4.33 & -2.69 \\
\hline Anterior insula/superior temporal gyrus & $\mathrm{R}$ & 27 & 24 & 0 & 116 & -3.59 & -2.46 \\
\hline Superior and middle temporal gyrus & $\mathrm{R}$ & 57 & -51 & 15 & 113 & -3.30 & -2.41 \\
\hline Amygdala/parahippocampal gyrus & $\mathrm{R}$ & 30 & 0 & -27 & 96 & -5.08 & -2.71 \\
\hline Dorsolateral prefrontal cortex precentral gyrus/middle frontal gyrus & $\mathrm{R}$ & 54 & 6 & 36 & 90 & -3.92 & -2.66 \\
\hline \multicolumn{8}{|c|}{ Correlations between pretreatment Stroop-effect related neural activity and cannabis-abstinence measures: } \\
\hline $\begin{array}{l}\text { Conjunction of pretreatment Stroop-related neural activity correlation with } \\
\text { three within-treatment cannabis-abstinence measures }{ }^{b}\end{array}$ & & & & & & $\mathbf{r}$ & $\mathbf{r}$ \\
\hline Occipital/lingual gyrus & $\mathrm{L}$ & -29 & -66 & I & 69 & 0.59 & 0.49 \\
\hline Posterior cingulate cortex & $L$ & -29 & -70 & 16 & 59 & 0.67 & 0.52 \\
\hline Occipital/Lingual Gyrus & $\mathrm{R}$ & 33 & -52 & -9 & 51 & 0.67 & 0.50 \\
\hline Dorsal anterior cingulate cortex & $\mathrm{R}$ & 3 & 3 & 38 & 22 & 0.59 & 0.49 \\
\hline Cuneus & $\mathrm{L}$ & -10 & -74 & 16 & 21 & 0.61 & 0.50 \\
\hline Supramarginal gyrus & $\mathrm{R}$ & 61 & -49 & 36 & 20 & 0.55 & 0.49 \\
\hline
\end{tabular}

Pretreatment Stroop-related neural activity correlation with \% days cannabis abstinence during I-year follow-up ${ }^{c}$

Positive correlations

Posterior cingulate/superior cerebellum

Occipital/fusiform gyrus

Occipital/fusiform gyrus

Ventral striatum/perigenual/subgenual anterior cingulate

Superior temporal/lateral orbitofrontal/inferior frontal gyrus

Occipital/fusiform/lingual gyrus/cerebellum

Negative correlations

Anterior insula/putamen /inferior frontal gyrus/caudate

$\begin{array}{rrrr}L & -3 & -66 & 9 \\ L & -24 & -90 & -15 \\ R & 42 & -78 & 0 \\ L & -6 & 30 & 12 \\ L & -39 & 18 & -21 \\ L & -24 & -51 & -15\end{array}$

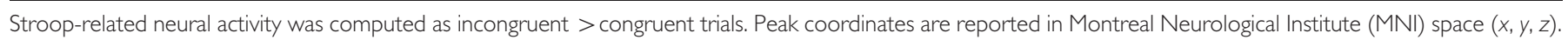
Cluster size is in $3 \times 3 \times 3 \mathrm{~mm}$ voxels.

a $t$-tests were computed to assess group differences between cannabis-dependent $(N=20)$ and healthy comparison $(N=20)$ individuals. Results are whole-brain family-wise-error (FWE) corrected for multiple comparisons at $p<0.05$.

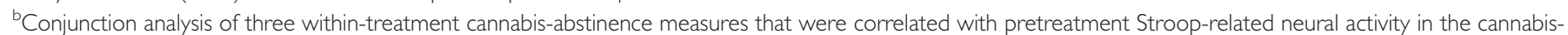
dependent group only $(N=20)$ : ( I) percent cannabis-negative urines, (2) percent self-reported cannabis-abstinent days during treatment, and (3) maximum selfreported consecutive days of abstinence (Table I for group means). All correlations were preformed robustly. Joint conjunction threshold is $p<0.000 \mathrm{I} 25$, with a cluster threshold of 20 voxels.

${ }^{c}$ A whole-brain robust correlation was computed to identify regions where Stroop-related neural activity was associated with long-term treatment outcome, as measured by percent of days of self-reported abstinence at I-year follow-up. Results are family-wise-error (FWE) corrected for multiple comparisons at $p<0.05$. Cannabis-dependent individuals with follow-up data available only, $\mathrm{N}=18$.

prefrontal cortex (although other regions showed greater activity; Gruber and Yurgelun-Todd, 2005). Others have also reported that amygdalar and hippocampal structure and function are altered in chronic cannabis users (Lorenzetti et al, 2010; Martin-Santos et al, 2010). The current findings are similar to those in other drug-abusing groups, eg, lower Stroop-related activity observed in stimulant dependence (Bolla et al, 2004; Nestor et al, 2011).

Stroop-related activity is thought to reflect a greater demand on cognitive-control mechanisms during incongruent relative to congruent trials. If this is the case, then-although we do not report performance differences in the current manuscript-the relative Stroop-related 

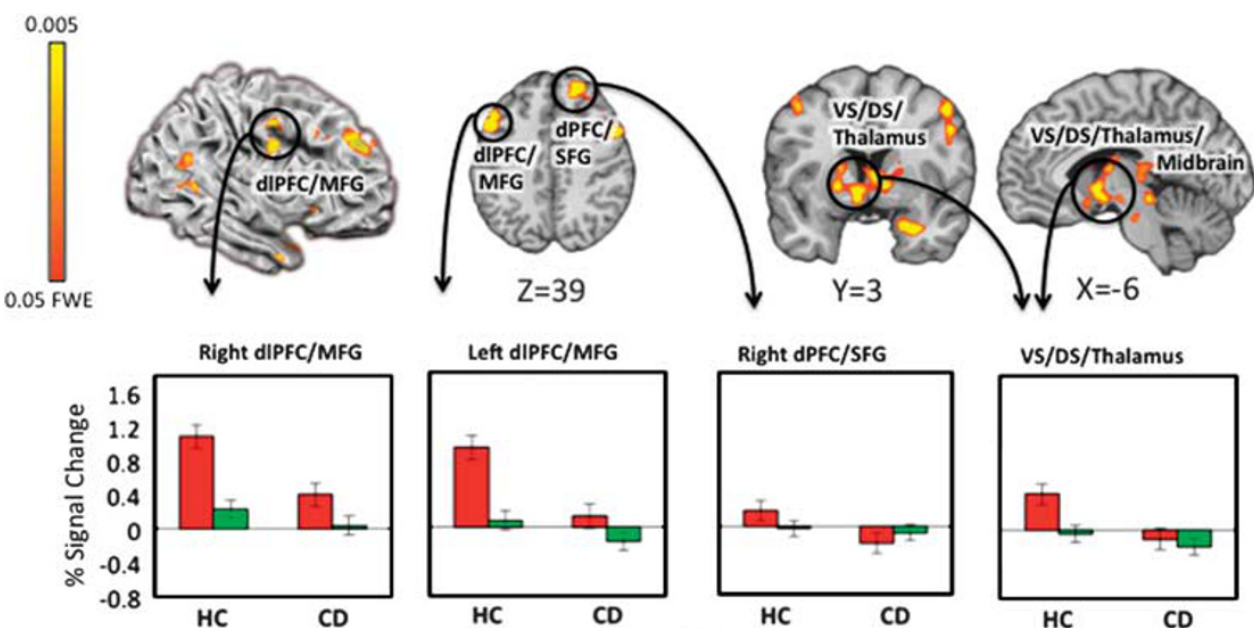

Left dIPFC/MFG

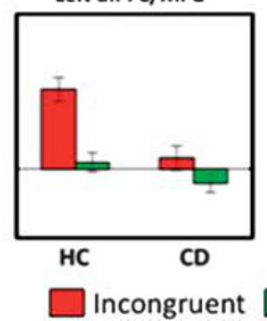

Right dPFC/SFG

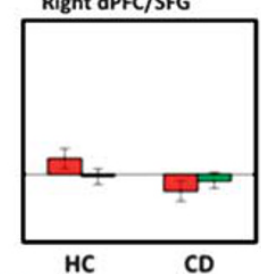

Congruent
VS/DS/Thalamus

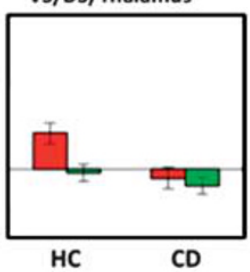

Figure I Differences in Stroop-related neural activity between cannabis-dependent and healthy-comparison individuals. Between-group differences in Stroop-related neural activity were computed as: healthy comparison (incongruent $>$ congruent) $>$ cannabis dependent (incongruent $>$ congruent). Bar graphs represent the extracted cluster-averaged percent signal change from incongruent and congruent trials ( \pm SEM). Results are family-wise-error corrected (FWE) at $p<0.05$. Left side of the brain is displayed on the left; $N=40$. Comparison subjects exhibited greater Stroop-related neural activity than cannabis-dependent subjects in regions of right dorsolateral prefrontal cortex (dIPFC), including middle frontal gyrus (MFG) and precentral gyrus; right dorsal prefrontal cortex (dPFC)/superior frontal gyrus (SFG) and left dIPFC/MFG; ventral striatum (VS), dorsal striatum (DS), thalamus, and midbrain. HC, healthy comparison; CD, cannabis dependent.
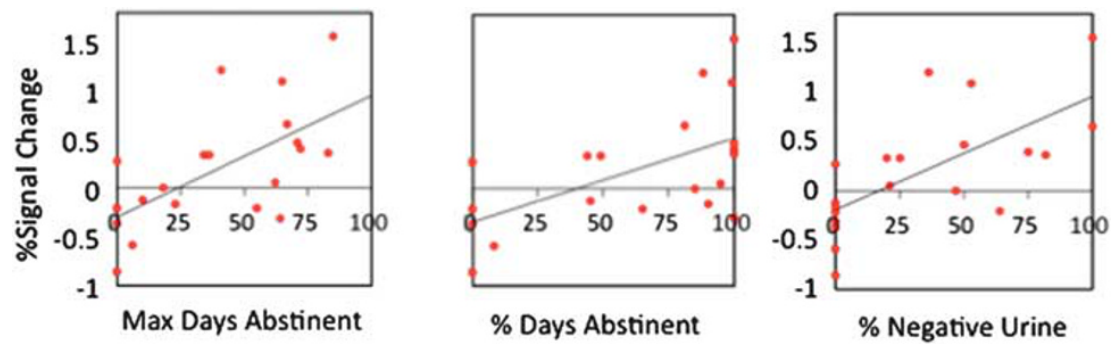

0.00000125
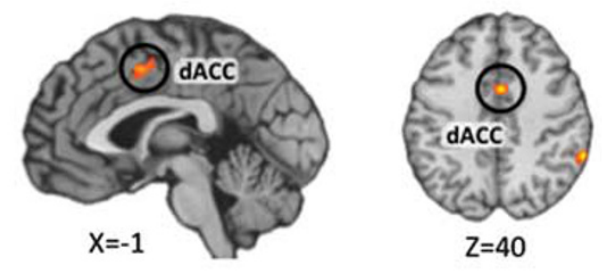

0.000125

Figure 2 Pretreatment Stroop-related neural activity correlated with three within-treatment abstinence measures in cannabis-dependent individuals. Robust correlations were computed between Stroop-related neural activity (incongruent $>$ congruent) and three different measures of within-treatment abstinence within the cannabis-dependent group $(N=20)$. Regions of overlap are shown at a conjunction threshold of $p<0.000$ I 25 , with a cluster threshold of $203 \times 3 \times 3 \mathrm{~mm}$ voxels. Scatter plots represent the extracted cluster-averaged percent signal change from Stroop-effect (incongruent $>$ congruent) contrast in overlap region of dorsal anterior cingulate cortex (dACC) identified in the conjunction. Left side of the brain is displayed on the left. Three variables, including maximum consecutive days of self-reported cannabis abstinence during treatment (max days abstinent), percent of days of self-reported cannabis abstinence during treatment (\% days abstinent), and percent of cannabis-negative urines during treatment (\% negative urine) were all correlated with Stroop-related neural activity in the dorsal anterior cingulate (cluster average $R^{2}=0.37,0.30$ and 0.42 , respectively).

hypo-activations may be interpreted as indicating reduced cognitive-control and salience detection in the substanceusing groups (Bhattacharyya et al, 2012; Bolla et al, 2004; Nestor et al, 2011). Importantly, each Stroop-sensitive subregion may implement specific subcomponents of cognitive control (Miller and Cohen, 2001; Peterson et al, 1999). For example, the anterior cingulate may implement conflict monitoring, vigilance, or error detection, whereas dorsolateral prefrontal cortex may contribute to control implementation and working memory and ventral striatum to salience detection (Bhattacharyya et al, 2012; Miller and Cohen, 2001).

The notions that lower activity reflects poorer cognitive control-as well as functional specificity-are supported by 

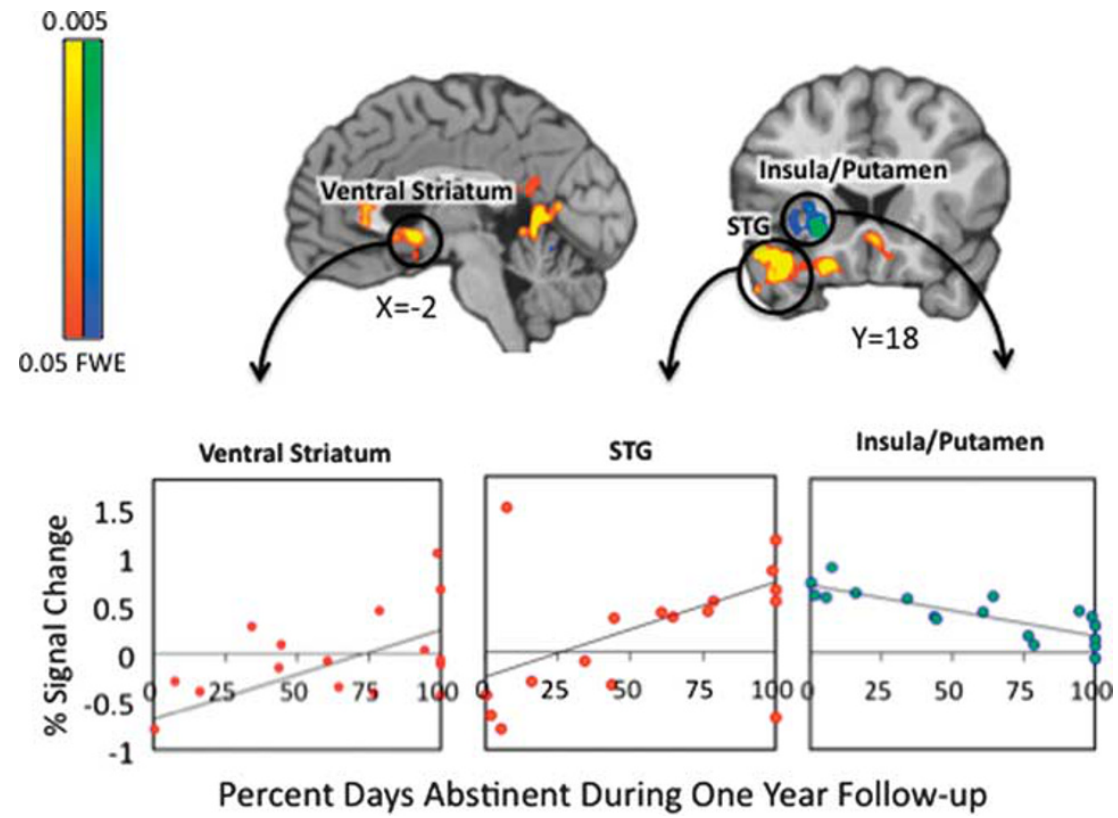

Figure 3 Pretreatment Stroop-related neural activity correlates with abstinence during I-year follow-up in cannabis-dependent individuals. Robust correlations were computed between Stroop-related neural activity (incongruent $>$ congruent) and percent of days of self-reported abstinence during I-year posttreatment follow-up period within cannabis-dependent individuals with available follow-up data $(\mathrm{N}=\mid 8)$. Results are family-wise-error corrected (FWE) at $p<0.05$. Scatter plots represent the extracted cluster-averaged percent signal change from Stroop-effect (incongruent $>$ congruent) contrast. Left side of the brain is displayed on the left. Stroop-related neural activity in ventral striatum correlated positively with abstinence rates during I-year follow-up (cluster average $R^{2}=0.39$ ). A similar relationship was seen in superior temporal gyrus (STG)/inferior frontal/lateral orbitofrontal gyrus (cluster average $R^{2}=0.79$ ). Conversely, Stroop-related neural activity in insula/putamen negatively correlated with abstinence during I-year follow-up (cluster average $\left.R^{2}=0.61\right)$.

findings that link reduced neural activity with lower cognitive-control task performance in both healthy adults (eg, Egner and Hirsch, 2005) and cannabis users (Hester et al, 2009; Wesley et al, 2011). For example, previous studies in cannabis users have shown that diminished anterior cingulate activation is related to diminished error awareness during Go/No-Go task performance (Hester et al, 2009) and suboptimal decision making during Iowa-Gambling-Task performance (Wesley et al, 2011). Cannabis users also show relative hypoactivity in the anterior cingulate during processing of affective faces (Gruber et al, 2009) and in the dorsal and ventral striatum during reward-anticipations (van Hell et al, 2010). Recently, Harding et al (2012) reported increased connectivity between prefrontal cortex and occipitoparietal regions during a cognitive-control interference task. In the absence of performance differences relative to matched controls, this was interpreted as compensatory disruptions in the neural processes underlying cognitive control. Similarly, others (Filbey and Yezhuvath, 2013) reported preliminary findings of greater connectivity between right frontal control network and substantia nigra/subthalamic nucleus in dependent compared with non-dependent cannabis users during successful response inhibition. Taken together, these data suggest a general pattern of disruption in cannabis users, which is often associated with neural hypoactivity and differences in cognitive processes (eg, Martin-Santos et al, 2010). In turn, this suggests reduced cognitive control and possibly reduced sensitivity to salient stimuli (eg, incongruent trials). However, the possibility that cannabisdependent individuals may be exhibiting greater neural efficiency during Stroop performance (eg, DeVito et al,
2012) is an alternate interpretation that warrants additional investigation.

\section{Correlations between fMRI Stroop and Cannabis-Use Outcomes}

We found positive associations between Stroop-related neural activity and within-treatment abstinence (dorsal anterior cingulate and lingual gyrus) and 1-year follow-up outcome (ventral striatum, inferior frontal gyrus, subgenual anterior cingulate and lingual gyrus) in regions typically activated during Stroop performance. Activity in these regions has previously been associated with cognitive control (dorsal anterior cingulate and inferior frontal gyrus), salience detection (ventral striatum) and processing of visual and language stimuli (eg, lingual gyrus). The idea that greater Stroop-related activity in these regions is beneficial or more normative is suggested by current and previous findings that: (1) healthy-comparison subjects show greater activity in these regions relative to cannabisdependent individuals (Eldreth et al, 2004); and (2) greater activity in these regions within drug-dependent groups is associated with better treatment outcome. For example, pretreatment Stroop-related fMRI activity in the prefrontal cortex and dorsal striatum was previously associated with less cocaine use during treatment (Brewer et al, 2008). The current findings extend this result to cannabis dependence and include measures of abstinence across a 1-year follow-up period. In addition, we have previously shown that Stroop-related activity in both prefrontal and subcortical (including midbrain) regions changes from preto posttreatment in cocaine dependence (DeVito et al, 
2012). Similarly, others have shown that increases in Stroop-related activity from pre- to posttreatment in midbrain and thalamic regions correlated with change in choice of cocaine stimuli in a laboratory task (Moeller et al, 2012). Therefore, Stroop-related activity in these regions may be a marker for better functioning, which may in turn predict better treatment response. Notably, activity in some regions correlated with both within- and posttreatment abstinence. Nevertheless, several regions correlated differentially with within- and posttreatment abstinence, which represent clinically distinct phases of treatment, with different challenges and contextual conditions. This adds to a growing literature relating neural activity to drug-use outcomes (including cannabis; eg, (Feldstein Ewing et al, 2013). Future studies could investigate whether treatmentphase-dependent factors, such as withdrawal severity and presence of structured support, are differentially associated with pretreatment cognitive-control-related neural activity.

\section{Limitations}

Although these data advance our understanding of the relationship between cognitive-control-related neural activity and cannabis treatment outcomes, we note several limitations. Some response-time data were missing because of technical failure, and therefore we cannot conclusively assess between-group differences in response times for the full sample for which fMRI data were available. Further, participants were scanned before treatment initiation, and treatment trial inclusion required active cannabis use within the past month. Thus, given cannabis' long half-life, patients may have had active cannabis in their systems or experienced withdrawal during scanning, and this may have influenced brain activity (Bhattacharyya et al, 2012). We addressed this limitation by including self-reported days of cannabis use in the month before treatment-onset (and fMRI scanning) and lifetime years of cannabis use as covariates in a re-analysis of the correlations between pretreatment Stroop effect and results were not substantially altered. Another limitation is that cannabis-dependent participants were all male, and nearly all were referred by the justice system. However, this is consistent with the demographic characteristics of cannabis users; prevalence of cannabis use is $\sim 2$ times as high among males than females, and $\sim 4$ times as high among individuals on probation or parole relative to the general population (SAMHSA, 2011). This suggests that this sample is representative of many treatment-seeking cannabis users. Similarly, higher rates of cigarette smoking and psychiatric conditions and lower IQ in the cannabis-dependent relative to the healthy-comparison group may have contributed to group differences; nevertheless, these differences are also representative of other samples of heavy cannabis users (eg, APA, 2013; Solowij et al, 2002). Future studies should assess whether these findings extend to females and to cannabis users not involved in the criminal justice system. Socioeconomic status was not assessed. Finally, the sample size was insufficient for analysis of differential effects of treatment subgroup. Future studies could assess whether Stroop-related neural activity differentially predicts treatment outcome across types of psychotherapy or pharmacotherapy. Nevertheless, this first study linking pretreatment cognitive-control neural activity with both within-treatment and longer-term abstinence advances our understanding of these processes considerably.

\section{Conclusions and Future Directions}

The current findings provide novel evidence in support of the hypothesis that cognitive-control-related neural processes are important not only for the initiation and maintenance of substance use, but also for achieving and maintaining abstinence (DeVito et al, 2012; Potenza et al, 2011; Streeter et al, 2008). However, the differential pattern of associations between pretreatment task-related neural activity and within-treatment $v s$ longer-term abstinence rates is novel and noteworthy. Given the aforementioned roles of different regions in cognitive control and Stroop performance, separable subcomponents of these neural systems (and the cognitive-affective processes they subserve) may be important for initial cannabis-use reduction in the context of outpatient treatment $v s$ 1-year after formal therapy has ended. These findings provide insight into potential biological mechanisms that might distinguish within-treatment and posttreatment cannabis-use outcomes and may account for current clinical challenges in predicting longer-term clinical outcomes from within-treatment abstinence indices (Carroll et al, under review; Peters et al, 2011). For example, the current findings are consistent with the possibility that relapse during different temporal epochs may be driven by different cognitive-control-related neural processes. These findings may therefore guide treatmentadaptation efforts and/or psychoeducational efforts to enhance efficacy and durability of treatment effects, although this possibility currently remains speculative.

To test this hypothesis directly, future studies could use neuroimaging at multiple time points (before/within/ following treatment) to examine the mechanisms underlying treatment durability, and specifically compare different types of therapies that exert their effects via different mechanisms (Potenza et al, 2011). For example, cognitivebehavioral therapy, a treatment with particularly durable and sometimes late-emerging therapeutic efficacy, may demonstrate brain-related differences in mechanisms of action from other behavioral therapies (eg, contingency management) that are associated shorter-term benefits and may rely less explicitly on cognitive control. Furthermore, as stepped interventions are receiving support for treating addictions (Drummond et al, 2009), the integration of brain-imaging measures at multiple time points might clarify the mechanisms underlying behavioral change in populations with different responses to different stages of stepped treatments.

The causes of the reported between-group differences in this study are not known. Indeed, any cognitive-control or neural-system disruptions in cannabis-dependent individuals may reflect pre-existing vulnerabilities for addiction, consequences of cannabis use, or a combination thereof. Regardless of cause, a compelling hypothesis is that these disruptions may be at least partially reversible with prolonged abstinence or treatment. Clinically, the regulation of craving involves cognitive control and recruitment of dorsolateral prefrontal cortex (Kober et al, 2010), in 
which activity negatively correlates with craving (Cousijn et al, 2012; Kober et al, 2010). In addition, response inhibition (a component of cognitive control) and its neural underpinnings may be modifiable with monetary incentives in adolescent cannabis users (Chung et al, 2011), suggesting that these skills and their underlying neural functions may be trainable and adaptable. Furthermore, activity in several neural regions associated with cannabis-use outcomes herein have previously been shown to be functionally altered following treatment (DeVito et al, 2012) or differ with longer abstinence duration (Connolly et al, 2012). As such, cognitive control, and its neural underpinnings, may serve as a marker of treatment success or a treatment target in part because of its impact on craving regulation, a key component of many successful, empirically validated treatments for addiction (eg, CBT; Carroll, 1998). In this light, the current findings suggest that 'normalization' of such neural markers (or the cognitive processes they subserve) may improve within-treatment outcomes, protect against later relapse, and/or improve rates of longer-term outcomes. These findings further suggest that successful periods of abstinence, cognitive-behavioral skill building, or regulation-of-craving training before (or early in) treatment may allow the partially recovered and strengthened brain to more effectively engage with subsequent treatment components or to overcome challenges (eg, resist cravings), which may lead to relapse. Future research should investigate whether these mechanisms, within the context of a more iterative, staged therapeutic process, result in greater short- and long-term improvements in cannabis-treatment outcomes (Budney et al, 2007).

\section{FUNDING AND DISCLOSURE}

This research was supported by P50-DA09241 and R01DA020908 from the National Institute on Drug Abuse (NIDA). HK is supported by K12 DA00167 from NIDA and UL1 RR024139 from the National Center for Research Resources. EED is supported by BIRCWH K12DA031050 from NIDA, National Institute on Alcohol Abuse and Alcoholism, Office of Research on Women's Health, and National Institutes of Health Office of the Director. HK, $\mathrm{EED}, \mathrm{CMD}$, and KMC report no financial relationships with commercial interests. MNP reports no financial conflict of interest with respect to the content of this manuscript, yet has received financial support or compensation for the following: consulted for and advised Boehringer Ingelheim, Lundbeck, and Ironwood; consulted for and has financial interests in Somaxon; received research support from the National Institutes of Health, Veteran's Administration, Mohegan Sun Casino, the National Center for Responsible Gaming and its affiliated Institute for Research on Gambling Disorders, and Forest Laboratories, OrthoMcNeil, Oy-Control/Biotie, Glaxo SmithKline, and Psyadon pharmaceuticals; participated in surveys, mailings, or telephone consultations related to drug addiction, impulse-control disorders, or other health topics; consulted for law offices and the federal public defender's office in issues related to impulse-control disorders; and provides clinical care in the Connecticut Department of Mental Health and Addiction Services Problem Gambling Services Program.

\section{ACKNOWLEDGEMENTS}

We would like to thank Charla Nich and Karen Hunkele for their valuable contribution to data management, particularly with the preparation of the clinical variables; Dan Marino, Scott Bullock, and Monica Solorzano for their subject recruitment and neuroimaging data collection; Maggie Mae Mell and Jiansong Xu for their assistance with the Stroop behavioral data; and Joanne Corvino and Iris Balodis for their help with managing recruitment efforts. We also thank all the participants for their time.

\section{REFERENCES}

APA (2013): Diagnostic and Statistical Manual of Mental Disorders-5th edn.

Battisti RA, Roodenrys S, Johnstone SJ, Pesa N, Hermens DF, Solowij N (2010). Chronic cannabis users show altered neurophysiological functioning on Stroop task conflict resolution. Psychopharmacology 212: 613-624.

Berkman ET, Falk EB, Lieberman MD (2011). In the trenches of real-world self-control: neural correlates of breaking the link between craving and smoking. Psychol Sci 22: 498-506.

Bhattacharyya S, Crippa JA, Allen P, Martin-Santos R, Borgwardt S, Fusar-Poli P et al (2012). Induction of psychosis by Delta9-tetrahydrocannabinol reflects modulation of prefrontal and striatal function during attentional salience processing. Arch Gen Psychiatry 69: 27-36.

Bolla K, Ernst M, Kiehl K, Mouratidis M, Eldreth D, Contoreggi C et al (2004). Prefrontal cortical dysfunction in abstinent cocaine abusers. J Neuropsychiatry Clin Neurosci 16: 456-464.

Brewer JA, Worhunsky PD, Carroll KM, Rounsaville BJ, Potenza MN (2008). Pretreatment brain activation during Stroop task is associated with outcomes in cocaine-dependent patients. Biol Psychiatry 64: 998-1004.

Budney AJ, Roffman R, Stephens RS, Walker D (2007). Marijuana dependence and its treatment. Addict Sci Clin Pract 4: 4-16.

Carpenter KM, Schreiber E, Church S, McDowell D (2006). Drug Stroop performance: relationships with primary substance of use and treatment outcome in a drug-dependent outpatient sample. Addict Behav 31: 174-181.

Carroll KM (1998). Therapy Manuals for Drug Addiction. A Cognitive-Behavioral Approach: Treating Cocaine Addiction. National Institute of Drug Abuse: Bethesda, MD, USA.

Carroll KM, Kiluk BD, Ball SA, Nich C (under review). Towards empirical identification of a valid, reliable and clinically meaningful indicator of treatment outcome for cocaine dependence.

Carroll KM, Kiluk BD, Nich C, Babuscio TA, Brewer JA, Potenza $\mathrm{MN}$ et al (2011). Cognitive function and treatment response in a randomized clinical trial of computer-based training in cognitive-behavioral therapy. Subst Use Misuse 46: 23-34.

Carroll KM, Kiluk BD, Nich C, DeVito EE, Decker S, Lapaglia D et al (2014). Toward empirical identification of a clinically meaningful indicator of treatment outcome: Features of candidate indicators and evaluation of sensitivity to treatment effects and relationship to one year follow up cocaine use outcomes. Drug Alcohol Depend 137C: 3-19.

Carroll KM, Nich C, Lapaglia DM, Peters EN, Easton CJ, Petry NM (2012). Combining cognitive behavioral therapy and contingency management to enhance their effects in treating cannabis dependence: less can be more, more or less. Addiction 107: 1650-1659.

Chung T, Geier C, Luna B, Pajtek S, Terwilliger R, Thatcher D et al (2011). Enhancing response inhibition by incentive: comparison of adolescents with and without substance use disorder. Drug Alcohol Depend 115: 43-50. 
Connolly CG, Foxe JJ, Nierenberg J, Shpaner M, Garavan H (2012). The neurobiology of cognitive control in successful cocaine abstinence. Drug Alcohol Depend 121: 45-53.

Cousijn J, Goudriaan AE, Ridderinkhof KR, van den Brink W, Veltman DJ, Wiers RW (2012). Neural responses associated with cue-reactivity in frequent cannabis users. Addict Biol 18: 570-580.

DeVito EE, Worhunsky PD, Carroll KM, Rounsaville BJ, Kober H, Potenza MN (2012). A preliminary study of the neural effects of behavioral therapy for substance use disorders. Drug Alcohol Depend 122: 228-235.

Drummond C, Coulton S, James D, Godfrey C, Parrott S, Baxter J et al (2009). Effectiveness and cost-effectiveness of a stepped care intervention for alcohol use disorders in primary care: pilot study. Br J Psychiatry 195: 448-456.

Egner T, Hirsch J (2005). The neural correlates and functional integration of cognitive control in a Stroop task. Neuroimage 24: 539-547.

Eldreth DA, Matochik JA, Cadet JL, Bolla KI (2004). Abnormal brain activity in prefrontal brain regions in abstinent marijuana users. Neuroimage 23: 914-920.

Feldstein Ewing SW, McEachern AD, Yezhuvath U, Bryan AD, Hutchison KE, Filbey FM (2013). Integrating brain and behavior: evaluating adolescents' response to a cannabis intervention. Psychol Addict Behav 27: 510-525.

Filbey F, Yezhuvath U (2013). Functional connectivity in inhibitory control networks and severity of cannabis use disorder. $A m J$ Drug Alcohol Abu 39: 382-391.

First MB, Spitzer RL, Gibbon M, Williams JB (1996). Structured Clinical Interview for DSM-IV Axis I Disorders-Patient Edition. Biometrics Research Department, New York State Psychiatric Institution: New York, NY, USA.

MTP Research Group (2004). Brief treatments for cannabis dependence: findings from a randomized multisite trial. $J$ Consult Clin Psychol 72: 455-466.

Gruber SA, Rogowska J, Yurgelun-Todd DA (2009). Altered affective response in marijuana smokers: an FMRI study. Drug Alcohol Depend 105: 139-153.

Gruber SA, Yurgelun-Todd DA (2005). Neuroimaging of marijuana smokers during inhibitory processing: a pilot investigation. Brain Res Cogn Brain Res 23: 107-118.

Harding IH, Solowij N, Harrison BJ, Takagi M, Lorenzetti V, Lubman DI et al (2012). Functional connectivity in brain networks underlying cognitive control in chronic cannabis users. Neuropsychopharmacology 37: 1923-1933.

Hester R, Nestor L, Garavan H (2009). Impaired error awareness and anterior cingulate cortex hypoactivity in chronic cannabis users. Neuropsychopharmacology 34: 2450-2458.

Kober H, Mende-Siedlecki P, Kross EF, Weber J, Mischel W, Hart CL et al (2010). Prefrontal-striatal pathway underlies cognitive regulation of craving. Proc Natl Acad Sci USA 107: 14811-14816.

Lorenzetti V, Lubman DI, Whittle S, Solowij N, Yücel M (2010). Structural MRI findings in long-term cannabis users: what do we know? Subst Use Misuse 45: 1787-1808.

MacLeod CM (1991). Half a century of research on the Stroop effect: an integrative review. Psychol Bull 109: 163-203.

Martin-Santos R, Fagundo A, Crippa J, Atakan Z, Bhattacharyya S, Allen $P$ et al (2010). Neuroimaging in cannabis use: a systematic review of the literature. Psychol Med 40: 383-398.

Miller EK, Cohen JD (2001). An integrative theory of prefrontal cortex function. Annu Rev Neurosci 24: 167-202.

Moeller SJ, Tomasi D, Woicik PA, Maloney T, Alia-Klein N, Honorio J et al (2012). Enhanced midbrain response at 6-month follow-up in cocaine addiction, association with reduced drugrelated choice. Addict Biol 17: 1013-1025.

Nestor L, McCabe E, Jones J, Clancy L, Garavan H (2011). Differences in 'bottom-up' and 'top-down' neural activity in current and former cigarette smokers: Evidence for neural substrates which may promote nicotine abstinence through increased cognitive control. Neuroimage 56: 2258-2275.

Peters EN, Nich C, Carroll KM (2011). Primary outcomes in two randomized controlled trials of treatments for cannabis use disorders. Drug Alcohol Depend 118: 408-416.

Peterson BS, Skudlarski P, Gatenby JC, Zhang H, Anderson AW, Gore JC (1999). An fMRI study of Stroop word-color interference: evidence for cingulate subregions subserving multiple distributed attentional systems. Biol Psychiatry 45: 1237-1258.

Potenza MN, Sofuoglu M, Carroll KM, Rounsaville BJ (2011). Neuroscience of behavioral and pharmacological treatments for addictions. Neuron 69: 695-712.

Robinson SM, Sobell LC, Sobell MB, Leo GI (2012). Reliability of the timeline followback for cocaine, cannabis, and cigarette use. Psychol Addict Behav 1-9.

SAMHSA (2011): Substance Abuse and Mental Health Services Administration. Results from the 2010 National Survey on Drug Use and Health: Summary of National Findings.

Schreiner AM, Dunn ME (2012). Residual effects of cannabis use on neurocognitive performance after prolonged abstinence: a meta-analysis. Exp Clin Psychopharmacol 20: 420-429.

Smith D, Jones P, Bullmore E, Robbins T, Ersche K (2013). Cognitive control dysfunction and abnormal frontal cortex activation in stimulant drug users and their biological siblings. Transl Psychiatry 3: 1-9.

Sobell LC, Sobell MB (1992). Timeline follow-back: a technique for assessing self-reported alcohol consumption. In: Litten RZAllen J (eds) Measuring Alcohol Consumption: Psychosocial and Biochemical Methods. Humana Press: Totowa, NJ, USA, pp 41-72.

Solowij N, Stephens RS, Roffman RA, Babor T, Kadden R, Miller M et al (2002). Cognitive functioning of long-term heavy cannabis users seeking treatment. JAMA 287: 1123-1131.

Stanger C, Elton A, Ryan SR, James GA, Budney AJ, Kilts CD (2013). Neuroeconomics and adolescent substance abuse: individual differences in neural networks and delay discounting. I Am Acad Child Adolesc Psychiatry 52: 747-755.

Stanger C, Ryan SR, Fu H, Landes RD, Jones BA, Bickel WK et al (2012). Delay discounting predicts adolescent substance abuse treatment outcome. Exp Clin Psychopharmacol 20: 205-212.

Streeter CC, Terhune DB, Whitfield TH, Gruber S, Sarid-Segal O, Silveri MM et al (2008). Performance on the Stroop predicts treatment compliance in cocaine-dependent individuals. Neuropsychopharmacology 33: 827-836.

Takagi M, Lubman DI, Cotton S, Fornito A, Baliz Y, Tucker A et al (2011). Executive control among adolescent inhalant and cannabis users. Drug Alcohol Rev 30: 629-637.

van Hell HH, Vink M, Ossewaarde L, Jager G, Kahn RS, Ramsey NF (2010). Chronic effects of cannabis use on the human reward system: an fMRI study. Eur Neuropsychopharmacol 20: 153-163.

Wager TD, Keller MC, Lacey SC, Jonides J (2005). Increased sensitivity in neuroimaging analyses using robust regression. Neuroimage 26: 99-113.

Wesley MJ, Hanlon CA, Porrino LJ (2011). Poor decision-making by chronic marijuana users is associated with decreased functional responsiveness to negative consequences. Psychiatry Res 191: $51-59$.

Zachary RA (1991). The Manual of the Shipley Institute of Living Scale-Revised. Western Psychological Services: LA, USA.

Supplementary Information accompanies the paper on the Neuropsychopharmacology website (http://www.nature.com/npp) 